\title{
Development of In Vivo Predictive pH-Gradient Biphasic Dissolution Test for Weakly Basic Drugs: Optimization by Orthogonal Design
}

\author{
Xiaowei Fan ${ }^{1}$, Shengying Shi ${ }^{1}$, Junlin $\mathrm{He}^{1}$, Jia Deng ${ }^{2,3^{*}}$, Jingou Ji ${ }^{1^{*}}$ \\ ${ }^{1}$ College of Chemistry and Chemical Engineering, Chongqing University, Chongqing, China. \\ ${ }^{2}$ Chongqing Key Laboratory of Natural Products, College of Environment and Resources, Chongqing Technology and Business University, Chongqing, China. \\ ${ }^{3}$ Central Nervous System Drug Key Laboratory of Sichuan Province, Luzhou, Sichuan, China.
}

\section{ABSTRACT}

e-mail: 725_tiger@sina.com jiadeng2011@hotmail.com

The aim of this study was to develop a method for set up and optimization of a pH-gradient biphasic dissolution model by orthogonal test design in light of the correlation with published in vivo data of ketoconazole (KTZ). A pH-gradient biphasic dissolution test was designed with a sequential $\mathrm{pH}$-gradient in the aqueous phase to simulate stomach, duodenum, jejunum, and ileum, and the organic phase was added in simulated small intestine conditions. The model was optimized by orthogonal test design with three factors and three levels and correlating with the published pharmacokinetic data of pure drug. The optimized dissolution conditions were $30 \mathrm{rpm}, 100 \mathrm{~mL}$ of an organic volume, and pH 5.5, 6.5, and 6.8 in the $\mathrm{pH}$-gradient aqueous phase in USP apparatus 2 . Under these conditions, KTZ dissolution displayed a good linear relationship with in vivo absorption $\left(R^{2}=0.85\right)$. This study indicates that this methodology is feasible to develop an in vivo predictive dissolution test.

KEYWORDS: pH-gradient biphasic dissolution test, in vitro-in vivo relationship (IVIVR), weakly basic drug, predictive dissolution test, ketoconazole, dissolution

\section{INTRODUCTION}

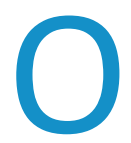

ral absorption of drugs is an extremely complicated process that is influenced by physiochemicalproperties of the drugformulation and physiological conditions of the gastrointestinal (GI) tract (1). Edwards in 1951 first proposed that drug dissolution would play a critical role in oral absorption, and this relationship has been of great interest in the past decades $(2,3)$. In vitro dissolution testing provides considerable information for in vivo drug performance by establishing an in vitro-in vivo relationship (IVIVR). Noticeably, when dissolution tests have inaccurate discrimination for the performance of candidate formulations between in vitro and in vivo studies, the predictive results may be completely misleading. Thus, it is desirable that changes in in vivo drug dissolution are reflected by the corresponding in vitro drug release test.

The oral absorption of Biopharmaceutics Classification System (BCS) class II drugs is mainly limited by solubility and dissolution, which may be required to establish the IVIVR $(4,5)$. In particular, weakly basic drugs are significantly influenced by physiological conditions in the $\mathrm{GI}$ tract, especially for dynamic $\mathrm{pH}$ conditions. Weak bases freely dissolve at gastric $\mathrm{pH}$, but not at intestinal $\mathrm{pH}$ due to a supersaturation-precipitation process that greatly affects drug bioavailability. Some dissolution tests may employ simple and single-pH non-physiologic buffers that do not accurately reflect in vivo dynamic situations $(6,7)$. To bridge the gap between in vitro and in vivo conditions, many attempts have been made to replicate the $\mathrm{Gl}$ process of weak bases by developing various biorelevant dissolution methods $(8,9)$. Owing to the lack of drug removal from the system and challenges associated with simulated absorption across the intestinal membrane, in vitro dissolution may overestimate the in vivo situation.

A biphasic dissolution test offers the advantage of maintaining sink conditions and having an absorptive phase, which has had increasing attention (10). A biphasic dissolution test can simulate drug dissolution and absorption in the $\mathrm{Gl}$ tract by the implementation of an immiscible organic phase acting as an absorptive sink over the aqueous solution. Moreover, the setup and handling of biphasic tests are relatively simple and cost 
effective, and drug release and partitioning are detected simultaneously within a single vessel (11). Previous studies have reported the development of various biphasic dissolution models and their correlation to pharmacokinetic (PK) data for different dosage forms (1217). A biphasic system with a single $\mathrm{pH}$ of aqueous phase was developed by combining United States Pharmacopeia (USP) apparatus 2 and 4 methods to discriminate three celecoxib formulations and obtain a consistent ranking between drug concentrations of organic phase and in vivo PK parameters (12). To better mimic drug behaviors in the $\mathrm{Gl}$ tract, a pH-adjusted biphasic dissolution system was developed to differentiate between four modified release formulations prepared with two weakly basic drugs (dipyridamole and BIMT 17) (13). The pH-adjusted biphasic test provided a ranking prediction with respect to in vivo absorption, but not in dissolution tests with different media at a constant $\mathrm{pH}$. However, there are still some questions to be considered and solved. Firstly, there is a lack of studies that develop a method for setup and optimization of a biphasic test because the experimental parameters may have a critical influence on drug performance $(11,18)$. Secondly, $\mathrm{pH}$-adjusted biphasic dissolution tests involve the addition of organic phase at gastric $\mathrm{pH}$, which might result in an overestimated prediction of in vivo performance due to supersaturation of weak bases, which could lead to overestimation of oral bioavailability if the organic phase is added prematurely.

Based on the aforementioned concerns, ketoconazole (KTZ), a BCS II weakly basic drug with broad-spectrum antifungal activity, was selected as a model drug. The aim of the present study was to develop a method to set up and optimize a $\mathrm{pH}$-gradient biphasic dissolution model by orthogonal test design in light of the correlation with published in vivo data of KTZ.

\section{MATERIALS AND METHODS}

\section{Materials}

KTZ was purchased from Wuhan Dahua Weiye Medicine Chemical Co., Ltd. (Wuhan, China). 1-octanol, hydrochloric acid $(\mathrm{HCl})$, sodium dihydrogen phosphate dihydrate, sodium hydroxide $(\mathrm{NaOH})$, and sodium chloride were obtained from Sichuan Kelun Pharmaceutical Co., Ltd. (Chengdu, China). Hard gelatin capsules (size 0) were donated by Suzhou Capsugel Ltd. (Suzhou, China). All other reagents used were of analytical grade.

\section{Solubility Determination}

Excessive $\mathrm{KTZ}$ was added to $10 \mathrm{~mL}$ of five different dissolution media (gastric buffer $\mathrm{pH}$ 2.0, phosphate buffer $\mathrm{pH} 5.5,6.5$, and 6.8 , and 1-octanol). The solubility test was performed at $37^{\circ} \mathrm{C}$ and $160 \mathrm{rpm}$ for $24 \mathrm{~h}$ using a horizontal shaker (THZ-98AB, Shanghai Yiheng Scientific Instrument Co. Ltd, Shanghai, China) $(n=3)$. Saturated solutions were filtered through a $0.45-\mu \mathrm{m}$ membrane filter. Drug concentration was measured by ultraviolet (UV) spectrophotometry at $224 \mathrm{~nm}$ (T6, Beijing Puxi General Instrument Co. Ltd, Beijing, China).

\section{pH-Gradient Biphasic Dissolution Test}

Considering the physiological $\mathrm{pH}$-change in the $\mathrm{Gl}$ tract which significantly influences KTZ dissolution, a $\mathrm{pH}$ gradient biphasic dissolution test was proposed (Fig. 1). The test was performed in USP apparatus 2 (RCZ-6B3, Shanghai Huanghai Pharmaceutical Inspection Instrument Co. Ltd, Shanghai, China) combining with a $\mathrm{pH}$ controlling device (PHS-2F, Shanghai INESA Scientific Instrument Co., Ltd, Shanghai, China).

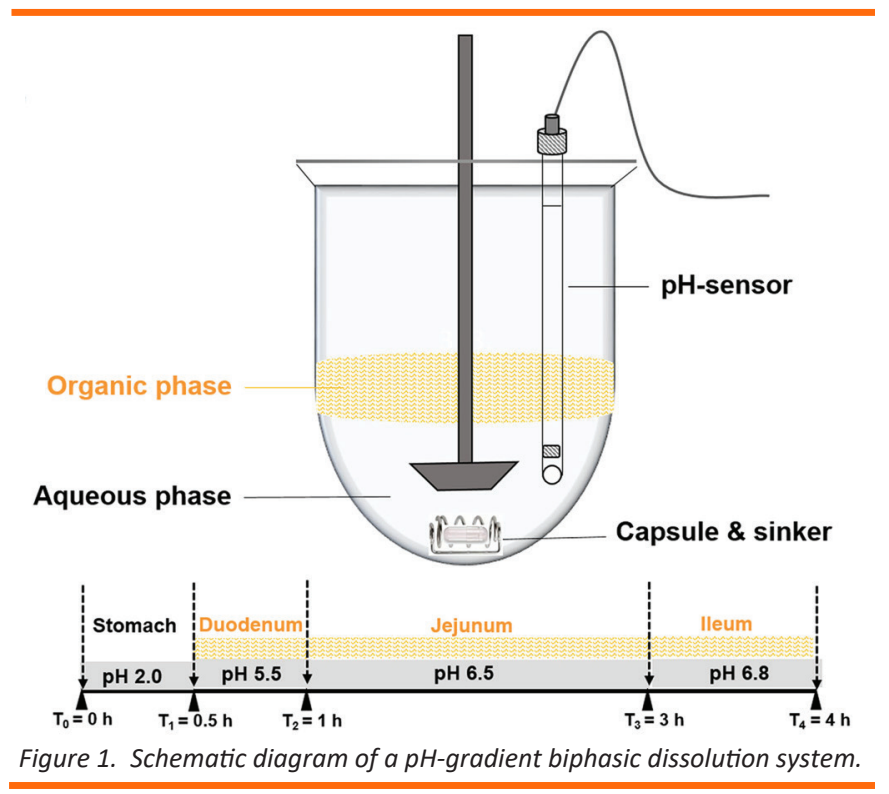

The gastric buffer was prepared with $250 \mathrm{~mL}$ of $0.01 \mathrm{~N}$ $\mathrm{HCl}$ with $0.1 \mathrm{M} \mathrm{NaCl}(\mathrm{pH} 2.0)$ and used to simulate drug dissolution in the stomach for $30 \mathrm{~min}$ (19). The $\mathrm{pH}$ of the aqueous medium was adjusted to duodenal $\mathrm{pH}$ by using $5 \mathrm{M} \mathrm{NaOH}$ with a fresh sampling needle, and 1-octonal as the upper organic phase was added to simulate the intestinal membrane as an absorptive sink. 1-octonal was saturated with water prior to use to keep a constant volume during the experiment. Subsequently, the $\mathrm{pH}$ of the aqueous phase was readjusted to 6.5 to mimic the jejunum for $2 \mathrm{~h}$, and then the final $\mathrm{pH}$ increased to 6.8 for $1 \mathrm{~h}$. The temperature was maintained at $37^{\circ} \mathrm{C}$.

For the dissolution test, $100 \mathrm{mg}$ of pure KTZ in a gelatin capsule was used with a sinker $(n=3)$; $3-\mathrm{mL}$ samples were withdrawn manually from the aqueous phases at $5,10,20,30,40,50,60,70,80,90,105,120,150,180$, 210, and $240 \mathrm{~min}$, while $2-\mathrm{mL}$ samples were collected 
in the organic phase at $40,50,60,70,80,90,105,120$, 150, 180, 210, and $240 \mathrm{~min}$. Each sample was replaced with the same volume of fresh media. Samples collected from the aqueous phase were passed through a $0.45-\mu \mathrm{m}$ membrane filter, and samples from the organic phase were centrifuged at 12,000 rpm for $20 \mathrm{~min}$ (TG-16, Gongyi Yuhua Instrument Co. Ltd, Gongyi, China). The drug concentration was determined by UV assaying at $224 \mathrm{~nm}$.

\section{Optimization Strategy}

To obtain an optimal dissolution model for good prediction of in vivo drug performance, optimization was carried out using a simple and efficient orthogonal experimental design. Rotating speed of the paddle (factor A), organic volume (factor $\mathrm{B}$ ), and $\mathrm{pH}$ gradients of the aqueous phase (factor C) are most likely to influence the dissolution process, so these were selected as three factors. The orthogonal experiment with three factors and three levels, as shown in Table 1, was first used for optimization of the biphasic dissolution test. Nine trials were performed based on the $L_{9}\left(3^{4}\right)$ matrix with a minimal number of trials by testing combinations (20). Correlation coefficient $\left(R^{2}\right)$ of a linear relationship between in vitro drug dissolution and the reported in vivo KTZ data obtained from (21) was selected as the evaluation index. The closer to 1 the $R^{2}$ value was, the better the predictive efficiency.

Table 1. Three Factors $(A, B$, and $C)$ and Three Levels of the Orthogonal Test Design

\begin{tabular}{|c|c|c|c|}
\hline Level & $\begin{array}{c}\text { A } \\
\text { (Rotating Speed, } \\
\text { rpm) }\end{array}$ & $\begin{array}{c}\text { B } \\
\text { (Organic Volume, } \\
\mathbf{m L}\end{array}$ & $\begin{array}{c}\text { C } \\
\text { (pH-Gradient } \\
\text { Aqueous Phase) }\end{array}$ \\
\hline 1 & 30 & 75 & $5.5,6.5,6.8$ \\
\hline 2 & 40 & 100 & $6.0,6.5,6.8$ \\
\hline 3 & 50 & 125 & $6.5,6.5,6.8$ \\
\hline
\end{tabular}

\section{Data Analysis}

All data were expressed as mean \pm standard deviation (SD). Linear regression analysis was used to evaluate the relationship between the percentage of drug release and the percentage of drug absorbed $\left(F_{a}\right)$ at corresponding time points. $F_{a}$ was calculated via Microsoft Excel 2013 (Redmond, WA, USA) based on the reported in vivo data from female rats using the Wagner-Nelson method (21, 22):

$$
\mathrm{F}_{\mathrm{a}}=\left\lfloor\left(C_{(t)}+\mathrm{k}_{e} \mathrm{AUC}_{(0-t)}\right) / \mathrm{k}_{e} \mathrm{AUC}_{(0-\mathrm{inf})}\right\rfloor \times 100
$$

In this equation, $F_{a}$ is the fraction of drug absorbed; $C_{(t)}$ is the drug concentration at time point $t ; \mathrm{k}_{\mathrm{e}}$ is the elimination rate constant; $\mathrm{AUC}_{0-t}$ is the area under the plasma concentration-time curve from zero to time $t$; and $A \cup C_{0 \text {-inf }}$ is the area under the curve from zero to infinity.

\section{RESULTS AND DISCUSSION}

\section{Solubility}

KTZ showed highly pH-dependent solubility in the physiologically relevant $\mathrm{pH}$ range (Table 2 ). As a weakly basic drug, KTZ had the highest solubility in the acidic media, with $8.2 \mathrm{mg} / \mathrm{mL}$ dissolved in $\mathrm{pH} 2.0$, which was used to mimic $\mathrm{pH}$ conditions of a healthy fasted stomach. In contrast, KTZ showed a dramatic decrease in solubility to $9.5 \mu \mathrm{g} / \mathrm{mL}$ in pH 5.5 buffer, following a slow fall from 3.9 to $3.5 \mu \mathrm{g} / \mathrm{mL}$ between $\mathrm{pH} 6.5$ and $\mathrm{pH} 6.8$. KTZ had high solubility of $5.6 \mathrm{mg} / \mathrm{mL}$ in 1-octanol due to its hydrophobic structure (logP 3.9) (23).

Table 2. Solubility of Ketoconazole in Dissolution Media at $37^{\circ} \mathrm{C}$

\begin{tabular}{|c|c|}
\hline Media & Solubility, mean $\pm \mathrm{SD}(\boldsymbol{n}=\mathbf{3})$ \\
\hline 1-Octanol & $5.6 \pm 0.5 \mathrm{mg} / \mathrm{mL}$ \\
\hline Gastric buffer pH 2.0 & $8.2 \pm 0.2 \mathrm{mg} / \mathrm{mL}$ \\
\hline PBS pH 5.5 & $9.5 \pm 0.5 \mu \mathrm{g} / \mathrm{mL}$ \\
\hline PBS pH 6.5 & $3.9 \pm 0.6 \mu \mathrm{g} / \mathrm{mL}$ \\
\hline PBS pH 6.8 & $3.5 \pm 0.6 \mu \mathrm{g} / \mathrm{mL}$ \\
\hline
\end{tabular}

PBS: phosphate buffer solution.

\section{Optimization Study}

The orthogonal experimental design was used to select the optimum dissolution conditions. The results of nine groups of experiments under different conditions are shown in Table 3. The $k\left(k_{1}, k_{2}\right.$, or $\left.k_{3}\right)$ value was the mean of the sum of the three $R^{2}$ values for levels $1-3$ of each factor, which revealed the change of predictive efficiency. The effects of levels 1-3 on drug dissolution were factor A (rotating speed of paddle): $1>2>3$; factor B (organic volume): $2>1>3$; factor $\mathrm{C}$ ( $\mathrm{pH}$ gradients of aqueous phase): $1>3>2$. The range (R) was the difference between the maximum and minimum value of $k_{1}, k_{2}$, and $k_{3}$ in the same table column, which reflected the impact degree of different factors. The larger the $\mathrm{R}$ value of one factor, the greater the impact on drug dissolution and in vivo prediction. According to the $\mathrm{R}$ value in Table 3 , the order of three factors influencing drug dissolution and in vivo prediction was $C>B>A$. Therefore, the optimized dissolution parameters were $A_{1} B_{2} C_{1}$; namely, rotating speed is $30 \mathrm{rpm}$, the organic volume is $100 \mathrm{~mL}$, and the $\mathrm{pH}$-gradient aqueous phase is $\mathrm{pH} 5.5,6.5$, and 6.8.

\section{pH-Gradient Biphasic Dissolution Test}

To validate the prediction potential of the optimized dissolution test, KTZ dissolution was performed in the optimum pH-gradient biphasic dissolution test in triplicate. The dissolution profiles of pure drug are presented in Figure 2. Drug dissolution reached 100\% 


\begin{tabular}{|c|c|c|c|c|c|}
\hline Test No. & $\begin{array}{c}\text { A } \\
\text { (Rotating Speed, rpm) }\end{array}$ & $\begin{array}{c}\text { B } \\
\text { (Organic Volume, } \mathrm{mL} \text { ) }\end{array}$ & $\begin{array}{c}\mathrm{C} \\
\text { (pH-gradient aqueous } \\
\text { phase) }\end{array}$ & $\begin{array}{c}\text { D } \\
\text { Blank }\end{array}$ & $R^{2}$ \\
\hline 1 & 30 & 75 & $5.5,6.5,6.8$ & 1 & 0.81 \\
\hline 2 & 30 & 100 & $6.0,6.5,6.8$ & 2 & 0.65 \\
\hline 3 & 30 & 125 & $6.5,6.5,6.8$ & 3 & 0.63 \\
\hline 4 & 40 & 75 & $6.0,6.5,6.8$ & 3 & 0.66 \\
\hline 5 & 40 & 100 & $6.5,6.5,6.8$ & 1 & 0.73 \\
\hline 6 & 40 & 125 & $5.5,6.5,6.8$ & 2 & 0.65 \\
\hline 7 & 50 & 75 & $6.5,6.5,6.8$ & 2 & 0.64 \\
\hline 8 & 50 & 100 & $5.5,6.5,6.8$ & 3 & 0.83 \\
\hline 9 & 50 & 125 & $6.0,6.5,6.8$ & 1 & 0.44 \\
\hline $\mathrm{k}_{1}$ & 0.70 & 0.70 & 0.76 & & \\
\hline $\mathrm{k}_{2}$ & 0.68 & 0.74 & 0.58 & & \\
\hline $\mathrm{k}_{3}$ & 2.64 & 0.57 & 0.67 & & \\
\hline $\mathrm{R}$ & 0.06 & 0.16 & 0.18 & & \\
\hline OC & $A_{1}$ & $\mathrm{~B}_{2}$ & $\mathrm{C}_{1}$ & & \\
\hline
\end{tabular}

Note: $k_{1}, k_{2}$, and $k_{3}$ are mean values of the sum of level 1-3 for each factor $(A-C) ; R$ is the difference between maximum and minimum value of $k_{1}, k_{2}$, and $k_{3}$ in the same column; $R^{2}$ : correlation coefficient; OC: optimized condition. oxide.
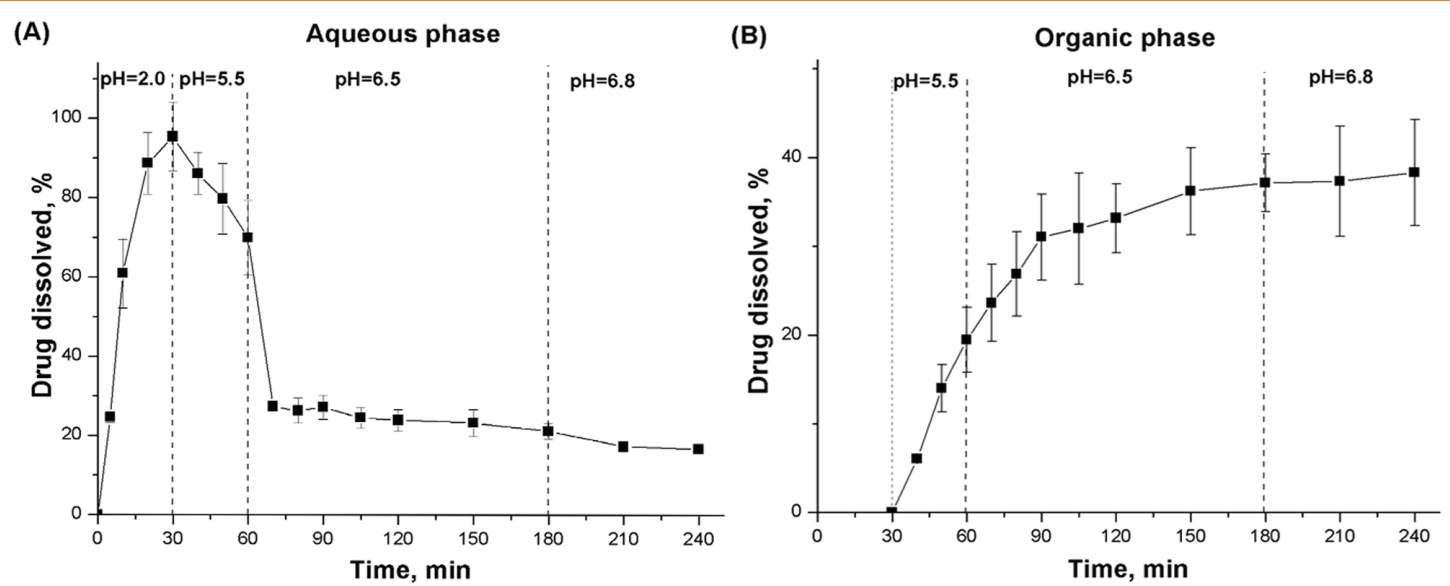

Figure 2. Dissolution profiles of pure ketoconazole in the optimized $\mathrm{pH}$-gradient biphasic dissolution test in the aqueous phase (A) and organic phase (B) (gastric buffer pH 2.0 for 30 min followed by $\mathrm{pH}$ adjustment to $5.5 \pm 0.05$ for $30 \mathrm{~min}, 6.5 \pm 0.05$ for $2 \mathrm{~h}$, and $6.8 \pm 0.05$ for $1 \mathrm{~h}$, respectively). Dashed vertical lines represents the $\mathrm{pH}$ changes.

in the gastric buffer $\mathrm{pH} 2.0$ due to high solubility in the acidic medium. After $\mathrm{pH}$ adjustment, drug concentration dramatically declined with increasing $\mathrm{pH}$ from 5.5 to 6.8 in the aqueous phase, even to below $20 \%$ in pH 6.8 medium (Fig. 2A). This could be attributed to rapid drug precipitation caused by low solubility of KTZ in the high $\mathrm{pH}$ media. In contrast, the amount of drug released gradually increased in the organic phase (Fig. 2B). It is well known that the small intestine is a main absorption site for most drugs (24). The addition of the organic phase after $\mathrm{pH}$ change was to simulate the intestinal absorption by removing dissolved drug from the aqueous phase and into the organic phase. Moreover, a good linear relationship was found between in vitro dissolution and in vivo $\mathrm{F}_{\mathrm{a}}\left(R^{2}=0.85\right)$ (Fig. 3), which was higher than the $R^{2}$ values obtained for all other conditions shown in Table 3 .

\section{CONCLUSION}

An orthogonal design using experimental data combined with reported in vivo data was used to set up and optimize a $\mathrm{pH}$-gradient biphasic dissolution test. The optimized experimental parameters were obtained based on $R^{2}$ of a linear relationship between in vitro and in vivo dissolution of KTZ. The highest $R^{2}$ was found when KTZ was tested in the optimized pH-gradient biphasic dissolution test, indicating feasibility of the method. In the next study, 
different KTZ formulations will be used to further identify the discriminative and predictive ability of the proposed biphasic dissolution test.

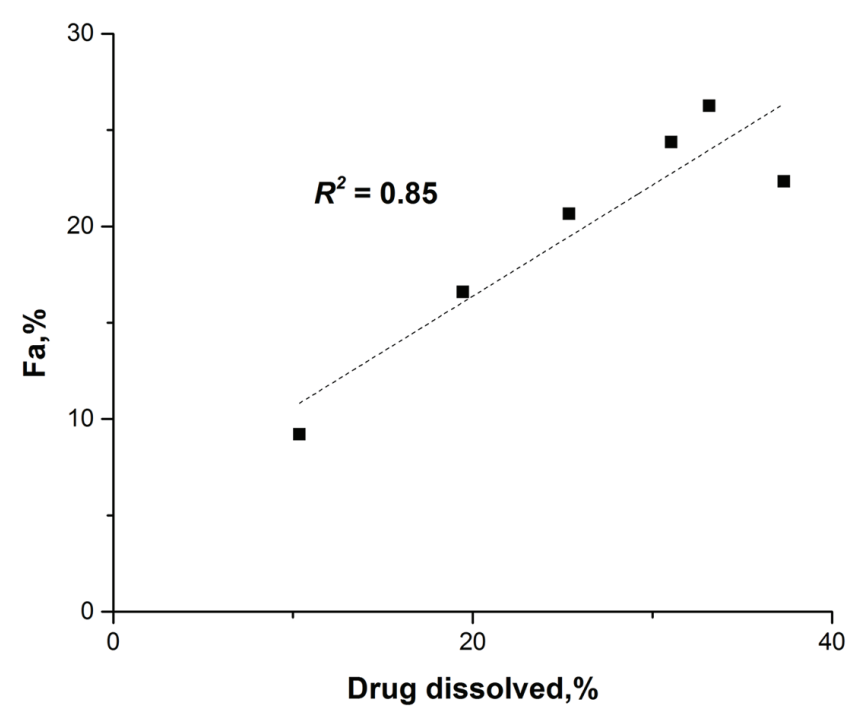

Figure 3. Relationship of in vitro dissolution of ketoconazole in the organic phase of optimized $\mathrm{pH}$-gradient biphasic test plotted against in vivo absorption (Fa) calculated according to data reported in female rats (21).

\section{FUNDING}

This work was financially supported for purchasing materials and assay determination by the Science and Technology Research Program of Chongqing Municipal Education Commission (Grant No. KJQN201800820); the Chongqing Research Program of Basic Research and Frontier Technology (Grant No. cstc2019jcyjmsxmX0753); the Scientific Research Project of Chongqing Technology and Business University (Grant No. 1951066); the open fund of the Key Laboratory of Natural Medicine Research of Chongqing Education Commission (Grant No. KFJJ2019093); the Open Project of Central Nervous System Drug Key Laboratory of Sichuan Province (Grant No. 200024-01SZ); and the Open Project of Central Nervous System Drug Key Laboratory of Sichuan Province (Grant No. 200024-01SZ).

\section{CONFLICT OF INTERESTS}

The authors disclosed no conflicts of interest related to this article.

\section{REFERENCES}

1. Jambhekar, S. S.; Breen, P. J. Drug dissolution: significance of physicochemical properties and physiological conditions. Drug Discov. Today. 2013, 18, 1173-1184. DOI: 10.1016/j. drudis.2013.08.013.

2. Edwards, L. J. The dissolution and diffusion of aspirin in aqueous media. Trans. Faraday Soc. 1951, 47, 1191-1210. DOI: 10.1039/ tf95 14701191.
3. Dokoumetzidis, A.; Macheras, P. A century of dissolution research: from Noyes and Whitney to the biopharmaceutics classification system. Int. J. Pharm. 2006, 321, 1-11. DOI: 10.1016/j.ijpharm.2006.07.011.

4. Amidon, G. L.; Lennernäs, H.; Shah, V. P.; Crison, J. R. A theoretical basis for a biopharmaceutic drug classification: the correlation of in vitro drug product dissolution and in vivo bioavailability. Pharm. Res.1995, 12,413-420. DOI: 10.1023/A:1016212804288.

5. Butler, J.; Dressman, J. B. The developability classification system: Application of biopharmaceutics concepts to formulation development. J. Pharm. Sci. 2010, 99, 4940-4954. DOI: 10.1002/ jps.22217.

6. Mudie, D. M.; Amidon, G. L.; Amidon, G. E. Physiological parameters for oral delivery and in vitro testing. Mol. Pharm. 2010, 7, 1388-1405. DOI: 10.1021/mp100149j.

7. Park, K. Absence of in vivo-in vitro correlation in per-oral drug delivery. J. Control. Release 2014, 180, 150. DOI: 10.1016/j. jconrel.2014.03.020.

8. Kostewicz, E. S.; Wunderlich, M.; Brauns, U.; Becker, R.; Bock, T.; Dressman, J. B. Predicting the precipitation of poorly soluble weak bases upon entry in the small intestine. J. Pharm. Pharmacol. 2004, 56, 43-51. DOI: 10.1211/0022357022511.

9. Butler, J.; Hens, B.; Vertzoni, M.; Brouwers, J.; Berben, P.; Dressman, J.; Andreas, C. J.; Schaefer, K J.; Mann, J.; McAllister, M.; Jamei, M.; Kostewicz, E.; Kesisoglou, F.; Langguth, P.; Minekus, M.; Mullertz, A.; Schilderink, R.; Koziolek, M.; Jedamzik, P.; Weitschies, W.; Reppas, C.; Augustijns, P. In vitro models for the prediction of in vivo performance of oral dosage forms: Recent progress from partnership through the IMI OrBiTo collaboration. Eur. J. Pharm. Biopharm. 2019, 136, 70-83. DOI: 10.1016/j.ejpb.2018.12.010.

10. Pestieau, A.; Evrard, B. In vitro biphasic dissolution tests and their suitability for establishing in vitro-in vivo correlations: A historical review. Eur. J. Pharm. Sci. 2017, 102, 203-219. DOI: 10.1016/j.ejps.2017.03.019.

11. Locher, K.; Borghardt, J. M.; Frank, K. J.; Kloft, C.; Wagner, K. G. Evolution of a mini-scale biphasic dissolution model: Impact of model parameters on partitioning of dissolved API and modelling of in vivo-relevant kinetics. Eur. J. Pharm. Biopharm. 2016, 105, 166-175. DOI: 10.1016/j.ejpb.2016.06.008

12. Shi, Y.; Gao, P.; Gong, Y.; Ping, H. Application of a biphasic test for characterization of in vitro drug release of immediate release formulations of celecoxib and its relevance to in vivo absorption. Mol. Pharm. 2010, 7, 1458-1465. DOI: 10.1021/mp100114a.

13. Heigoldt, U.; Sommer, F.; Daniels, R.; Karl-Gerhard, W. Predicting in vivo absorption behavior of oral modified release dosage forms containing $\mathrm{pH}$-dependent poorly soluble drugs using a novel $\mathrm{pH}$-adjusted biphasic in vitro dissolution test. Eur. J. Pharm. Biopharm. 2010, 76, 105-111. DOI:10.1016/j.ejpb.2010. 05.006.

14. Deng, J.; Staufenbiel, S.; Hao, S.; Wang, B.; Dashevskiy, A.; Bodmeier, R. Development of a discriminative biphasic in vitro dissolution test and correlation with in vivo pharmacokinetic 
studies for differently formulated racecadotril granules. J. Control. Release. 2017, 255, 202-209. DOI: 10.1016/j. jconrel.2017.04.034.

15. Pestieau, A.; Lebrun, S.; Cahay, B.; Brouwers, A.; Streel, B.; Cardot, J. M.; Evrard, B. Evaluation of different in vitro dissolution tests based on level $A$ in vitro-in vivo correlations for fenofibrate selfemulsifying lipid-based formulations. Eur. J. Pharm. Biopharm. 2017, 112, 18-29. DOI: 10.1016/j.ejpb.2016.10.030.

16. Deng, J.; Staufenbiel, S.; Bodmeier, R. Evaluation of a biphasic in vitro dissolution test for estimating the bioavailability of carbamazepine polymorphic forms. Eur. J. Pharm. Sci. 2017, 105, 64-70. DOI:10.1016/j.ejps.2017. 05.013.

17. Xu, H.; Shi, Y.; Vela, S.; Marroum, P.; Gao, P. Developing quantitative in vitro-in vivo correlation for fenofibrate immediate-release formulations with the biphasic dissolutionpartition test Method. J. Pharm. Sci. 2018, 107, 476-487. DOI: 10.1016/j.xphs.2017.06.018.

18. Vangani, S.; Li, X. L.; Zhou, P.; Del-Barrio, M. A.; Chiu, R.; Cauchon, N.; Gao, P.; Medina, C.; Jasti, B. Dissolution of poorly water-soluble drugs in biphasic media using USP 4 and fiber optic system. Clin. Res. Regul. Aff. 2009, 26, 8-19. DOI: 10.1080/10601330902905887.
19. Klein, $\mathrm{S}$. The use of biorelevant dissolution media to forecast the in vivo performance of a drug. AAPS. J. 2010, 12, 397-406. DOI: 10.1208/s12248-010-9203-3.

20. Fan, W.; Zhu, W.; Zhang, X.; Di, L. The preparation of curcumin sustained-release solid dispersion by hot melt extrusion-I. Optimization of the formulation. J. Pharm. Sci. 2020, 109, 12421252. DOI: 10.1016/j.xphs.2019.11.019.

21. Zhou, Y.; He, P.; Liu, A.; Zhang, L.; Liu, Y. D.; Dai, R. Drug-drug interactions between ketoconazole and berberine in rats: pharmacokinetic effects benefit pharmacodynamic synergism. Phytother. Res. 2012, 26, 772-777. DOI: 10.1002/ptr.3621.

22. Takka, S.; Sakr, A.; Goldberg, A. Development and validation of an in vitro-in vivo correlation for buspirone hydrochloride extended release tablets. J. Control. Release. 2003, 88, 147-157. DOI:10.1016/S0168-365 9(02)00490-X.

23. Ghasemi, J.; Saaidpour, S. Quantitative structure-property relationship study of $n$-octanol-water partition coefficients of some of diverse drugs using multiple linear regression. Anal. Chim. Acta. 2007, 604, 99-106. DOI: 10.1016/j.aca.2007.10.004.

24. Murakami, T. Absorption sites of orally administered drugs in the small intestine. Expert. Opin. Drug Discov. 2017, 12, 1219-1232. DOI: 10.1080/17460441.2017.1378176. 